\title{
COVID-19 Vaccine Administration and Hesitation Among Psychiatric Emergency Services Patients
}

\author{
Lorena Mitchell $^{1}\left[\right.$ Meghan Wilkosz $^{1} \cdot$ Brian Fuehrlein $^{1,2}$
}

Received: 27 September 2021 / Accepted: 23 January 2022 / Published online: 12 February 2022

This is a U.S. government work and not under copyright protection in the U.S.; foreign copyright protection may apply 2022 , corrected publication 2022

\begin{abstract}
Psychiatric Emergency Services (PES) at Connecticut Department of Veterans Affairs (VACT) began offering the COVID19 vaccine to eligible veterans in February 2021. From February 10 to March 17, 2021 there were 110 encounters where a veteran was offered the vaccine (96 unique veterans). Of those 96 veterans, 39 (40.6\%) were interested in receiving the vaccine. Of those, $23(60.0 \%)$ veterans received the first dose of the Pfizer-BioNTech vaccine and among those, $21(91.3 \%)$ eventually received the second dose. Sixteen veterans were interested but unable to receive the vaccine due to PES-related obstacles. Common themes regarding vaccine hesitancy among this population include vaccine mistrust and concerns about side effects. Offering the vaccine to PES patients allowed VACT to reach a vulnerable subset of veterans who may be at higher risk of contracting the virus and experience worse disease outcomes.
\end{abstract}

Keywords COVID-19 $\cdot$ Vaccination $\cdot$ Veterans $\cdot$ Emergency $\cdot$ Mental health

\section{Introduction}

The Department of Veterans Affairs (VA) has implemented a national public health campaign to address the COVID-19 pandemic including providing COVID-19 vaccinations to all veterans. In December 2020, VA Connecticut (VACT) was one of 37 initial VA sites to offer the vaccine to employees and veterans. Based on risk stratification, the VA prioritization matrix, vaccine supply, and progression through other priority groups, VACT psychiatric emergency services (PES) began offering the vaccine to patients receiving its services in February 2021.

For many veterans with poor outpatient follow-up, the primary point of contact for medical care is the emergency room. VACT's PES serves patients who are potentially

Lorena Mitchell

Lorena.Mitchell@va.gov

Meghan Wilkosz

Meghan.Wilkosz@va.gov

Brian Fuehrlein

Brian.Fuehrlein@va.gov

1 Department of Veterans Affairs, Connecticut Healthcare System, 950 Campbell Ave, West Haven, CT 06516, USA

2 Department of Psychiatry, Yale University School of Medicine, 300 George St, New Haven, CT 06511, USA at elevated risk for contracting COVID-19. Most patients served by PES have substance use disorders (Elmarasi et al., 2021) which increases the risk of contracting COVID-19 (Wang et al., 2021). Individuals with substance use disorders have higher rates of COVID-19 related hospitalization and death (Wang et al., 2021). This group is also known to have vaccine hesitancy, with one recent study showing less than half felt immediately ready to receive the vaccine (Masson et al., 2021).

Beginning February 2021, all veterans who presented to PES were eligible for the COVID-19 vaccine. At that time, only the Pfizer-BioNTech vaccine was available to VACT veterans. This vaccine is a two-dose series approximately 21 days apart (U.S. Food and Drug Administration, 2021). As part of routine patient care, staff offered the vaccine, documented reasons for declining, obtained informed consent from those interested, and administered the first dose. PES staff also assisted with scheduling patients for their second dose and facilitating transportation back to the VA when necessary. 


\section{Methods}

Beginning in February 2021, the multidisciplinary PES team, with assistance from pharmacy, developed a procedure to offer and administer vaccines to veterans presenting to PES. To be eligible, the veteran had to be clinically sober and have capacity to consent to medical decision making. Additionally, the veteran had to be willing and able to return to the VA for the 2nd dose in approximately 21 days.

The inpatient pharmacy was responsible for vaccine allocation throughout the hospital. If a PES veteran was clinically appropriate and interested in receiving the vaccine, an order was sent to alert inpatient pharmacy. The Pfizer-BioNTech vaccine is prepared in increments of six doses. Once opened, all six doses must be administered within a 6-h time frame. Given these restrictions and concerns over waste, pharmacy would only open vials if all six doses could be administered in a timely manner. Hence, whether an interested PES veteran was able to be vaccinated depended on hospital wide vaccine dose allocation. If a dose was available, a PES nurse administered the vaccine and observed the veteran for $15 \mathrm{~min}$. The veteran was provided with a Pfizer-BioNTech COVID-19 vaccine EUA Fact Sheet for Recipients and Caregivers, a Center for Disease Control (CDC) vaccine record card, and a follow up appointment in approximately 21 days. If a veteran was interested and the dose was unavailable during their stay in PES, veterans were offered an outpatient appointment.

To ensure second dose compliance for those who received the first dose in PES, the PES social worker tracked all second dose appointments. Veterans were called approximately 2 days in advance of their vaccine appointment to offer a reminder and ensure transportation was available. The social worker was able to assist with rescheduling in cases where the veteran was unable to make the second dose appointment.

When veterans declined the vaccine, the PES social worker documented their refusal and reasoning in the electronic health record. Veterans were encouraged to follow up with their outpatient providers if they became interested at a later time. The study period ended in March 2021 when the majority of PES veterans were either vaccinated or had documented vaccination refusal in their health record.

\section{Results}

The study period was from February 10, 2021 to March 17, 2021. During this time, there were 117 total encounters. Seven veterans were unable to consent to vaccination due to severe symptoms of psychosis (4), intoxication (1), and confusion or delirium (2). This left 110 qualifying presentations and 96 unique veterans who were offered vaccination during the study period. Fourteen veterans presented to the PER more than once during the study period, all of whom initially declined the vaccine. They were offered the vaccine each time they presented and reasons for declining were documented each time. While none opted for the vaccine on subsequent PER visits, eight of these veterans were eventually vaccinated as an outpatient.

PES was able to provide the second vaccine dose to five veterans who received care around the time that their second dose was due. Two veterans did not follow up for the second dose as outpatients: one veteran has been lost to follow up with no contact with the VA since the PER visit; and the second veteran missed his appointment, although has been engaged in outpatient care so likely due to administrative oversight was not offered a second dose. Both veterans have primary diagnoses of an alcohol use disorder.

\section{Obstacles to Receiving the Vaccine in the ER}

For all veteran refusals (including those who presented more than once) the primary reason was documented. Out of 110 qualifying presentations, there were 87 encounters where a veteran did not receive a vaccine. Sixteen of these veterans were interested in the vaccine but unable to receive it during their stay in the PES. This was primarily due to the pharmacy being unable to allocate doses in a way to minimize waste and/or the veteran was transferred to an outside hospital inpatient unit prior to vaccination. If a veteran was transferred to the VA inpatient psychiatric unit, vaccination was available there. In three cases, a veteran was interested in receiving the vaccine, but it was contraindicated due to recent tetanus shot (2) and recent COVID-19 infection (1). Recent vaccination and COVID-19 infection are no longer considered contraindications for vaccination.

\section{Reasons for Vaccine Hesitancy}

Six veterans refused all care or did not provide a reason for declining vaccination. In these cases, the veterans' refusal was related to a broader pattern of behavior of refusing all care and may not correlate to views on vaccination. Fifteen veterans expressed feelings of mistrust or concern related specifically to the vaccine. For example, one veteran shared that he wanted to wait until full FDA approval rather than the current emergency use authorization (EUA). Another veteran felt strongly about the brand of vaccine being offered. Many veterans cited concerns about side effects and shared that they wanted to wait until more people had received the vaccine. In general, this group of veterans were open to receiving more information about the vaccine and remained open 
to receiving it in the future. Nineteen veterans expressed strong mistrust of the COVID-19 vaccine specifically and vaccines in general. For example, one veteran shared "I don't want to be part of the vaccine experiment" and another said "I don't get any vaccines. I don't want to put something in my body for something that I don't have and isn't a problem for me." Seventeen veterans declined the vaccine as they were more focused on their presenting concern to PES. Veterans in this group often were experiencing uncomfortable withdrawal symptoms and were focused on immediate relief and treatment. Five veterans shared they were not concerned about COVID-19 infection and thus declined the vaccine. Three of these veterans had recent COVID-19 infections. One veteran shared he was not concerned about COVID-19 because "it isn't a problem" for him.

Nine veterans declined the vaccine for reasons related to the emergency room setting. For example, five veterans requested discharge as soon as possible which made vaccine administration impossible due to logistical barriers. Another four veterans stated they preferred to get the vaccine outpatient and PES was able to assist them with scheduling an outpatient follow up appointment.

\section{Discussion}

VACT PES facilitated COVID-19 vaccinations for 23 veterans between February and March 2021. Of those, 21 veterans received the second dose and became fully vaccinated. The PES setting presented a number of obstacles to vaccination. As a point of triage, many veterans only stay briefly in PES before they are discharged or transferred to another setting for care. It proved challenging to secure and administer the vaccine to veterans who were discharging or transferring in a short amount of time. Veterans presenting to PES may also prioritize care for their emergent concern over addressing vaccination status for an ongoing pandemic. Additionally, VA PES often treats veterans who are intoxicated, or experiencing other acute psychiatric crises. In these cases, is not clinically appropriate to offer and administer the vaccine.

While there were challenges to offering and administering the vaccine in an emergency room setting, there were clear benefits too. VA Healthcare system in general serves a vulnerable subset of veterans who are more likely to be low income, belong to a minority group, be unemployed, and have a mental health disorder diagnosis (Agha et al., 2000; Eibner et al., 2016). Specifically, VA PES serves veterans who are not well engaged in outpatient and preventative care, have severe mental illness and substance use disorders, and may be living in congregate settings like homeless shelters or residential treatment facilities. By offering the vaccine in PES, we were able to reach a very vulnerable subset of veterans who may have not otherwise received that vaccine so quickly or at all.

When the VA PES began offering vaccines, only the Pfizer-BioNTech vaccination was available. Since then, Janssen has received emergency authorization for use. As the Janssen vaccine is a single dose, it may be more appropriate for the PES setting as patients are not required to follow up for a second dose. This is especially true for veterans who seek emergency care who are also experiencing homelessness, have severe substance use disorders, and other veterans who may have difficulty engaging in outpatient follow up. VA PES has administered three Janssen vaccine doses since the conclusion of this study.

Less than half of veterans offered the vaccine were interested in vaccination. This low interest is partly attributable to obstacles specific to the PES setting articulated above and partly to reasons specific to the COVID-19 vaccine and vaccines in general. This study tracked reasons for declining the vaccine during the first months of availability. It is possible that veterans who had reservations about vaccination at that time have since changed their mind and chosen to get vaccinated, as more information about vaccine safety and efficacy has become available.

Vaccination has become deeply politicized in the United States and thus a person's decision to be vaccinated may be related to their political, religious, class, or racial identity, and may be influenced by geographic location, employment, and education. A more in-depth qualitative exploration of these complicated and intersecting factors were beyond the scope of this study, but were undoubtedly at play as veterans considered vaccination during their emergency room visit.

Funding No funding was received to assist with the preparation of this manuscript.

\section{Declarations}

Conflict of interest The authors have no conflicts of interest to declare that are relevant to the content of this article.

Ethical Approval This study was approved by the VA Connecticut Healthcare System Human Research Protection Program.

\section{References}

Agha, Z., Lofgren, R. P., VanRuiswyk, J. V., \& Layde, P. M. (2000). Are patients at veterans affairs medical centers sicker? A comparative analysis of health status and medical resource use. Archives of Internal Medicine, 160(21), 3252-3257. https://doi.org/10.1001/ archinte.160.21.3252

Eibner, C., Krull, H., Brown, K. M., Cefalu, M., Mulcahy, A. W., Pollard, M., Shetty, K., Adamson, D. M., Amaral, E. F., Armour, P., 
Beleche, T., Bogdan, O., Hastings, J., Kapinos, K., Kress, A., Mendelsohn, J., Ross, R., Rutter, C. M., Weinick, R. M., Woods, D., ... Farmer, C. M. (2016). Current and Projected Characteristics and Unique Health Care Needs of the Patient Population Served by the Department of Veterans Affairs. Rand Health Quarterly, 5(4), 13.

Elmarasi, M., Garcia-Vassallo, G., Campbell, S., \& Fuehrlein, B. (2021). Brief report: Rates of fentanyl use among psychiatric emergency room patients. The American Journal on Addictions, 30, 92-95. https://doi.org/10.1111/ajad.13087

Masson, C. L., McCuistian, C., Straus, E., Elahi, S., Chen, M., Gruber, V. A., Le, T., \& Guydish, J. (2021). COVID-19 vaccine trust among clients in a sample of California residential substance use treatment programs. Drug and Alcohol Dependence, 225, 108812. https://doi.org/10.1016/j.drugalcdep.2021.108812

U.S. Food and Drug Administration. (September 2021). Fact sheet for healthcare providers administering vaccine-Emergency Use
Authorization (EUA) of the Pfizer-BioNTech COVID-19 vaccine to prevent coronavirus disease 2019. U.S Food and Drug Administration.

Wang, Q. Q., Kaelber, D. C., Xu, R., \& Volkow, N. D. (2021). COVID19 risk and outcomes in patients with substance use disorders: Analyses from electronic health records in the United States. Molecular Psychiatry, 26(1), 30-39. https://doi.org/10.1038/ s41380-020-00880-7

Publisher's Note Springer Nature remains neutral with regard to jurisdictional claims in published maps and institutional affiliations. 